\title{
CME Emergencies in gastroenterology (95278): self-assessment questionnaire
}

\author{
Edited by Adam Harris and Tahseen Chowdhury
}

\section{SAQs and answers are ONLINE for RCP fellows and collegiate members \\ The SAQs printed in the CME section can only be answered online to achieve external CPD credits. Any comments should be sent in via email only: clinicalmedicine@rcplondon.ac.uk}

\section{Format}

Candidates are asked to choose the best answer from the five possible answers. This best of five format is used in many medical examinations, however the questions are not intended to be representative of those used in the MRCP(UK) Part 1 or Part 2 Written Examinations.

\section{The answering process}

1 Go to www.rcplondon.ac.uk/SAQ

2 Log on using your usual RCP username and password

3 Select the relevant CME question paper

4 Answer all 10 questions by selecting the best answer from the options provided

5 Once you have answered all the questions, click on Submit

\section{Registering your external CPD credits}

Carrying out this activity allows you to claim two external CPD credits. These will be automatically transferred to your CPD diary, where you can review the activity and claim your points.

1 A 24-year-old woman, who was normally fit and well, presented to the emergency department with abdominal pain, nausea and vomiting. She was noted to have scleral jaundice and admitted to taking a paracetamol overdose 24 hours previously. Blood tests were sent. On examination, she appeared drowsy when trying to get a full history. An arterial blood gas revealed $\mathrm{pH}$ of 7.38 (7.35-7.45), plasma glucose $3.8 \mathrm{mmol} / \mathrm{L}$ (3.9-5.5) and serum lactate of $4.2 \mathrm{mmol} / \mathrm{L}(0.5-2.2)$.

\section{What will be your initial management?}

(a) await blood results to assess paracetamol levels before commencing treatment

(b) commence $\mathrm{N}$-acetylcysteine and intravenous fluids

(c) organise cerebral imaging

(d) referral to psychiatric liaison for review

(e) start lactulose
2 A 42-year-old ex-intravenous drug user presented with widespread lymphadenopathy and investigations revealed a diagnosis of lymphoma. Screening for HIV was negative. Hepatitis B and C screening revealed:

> hepatitis B surface antigen: negative

> hepatitis B surface antibody: positive

$>$ hepatitis B core antibody: positive

$>$ hepatitis B DNA: negative

$>$ hepatitis $C$ antibody: positive

> hepatitis C RNA: negative

Liver function tests were normal and imaging of the patient's abdomen revealed no evidence of chronic liver disease. Chemotherapy including high-dose steroids and rituximab was planned.

\section{Which of the following statements is correct?}

(a) Viral serology is consistent with active hepatitis $C$ infection and the patient may be at risk of acute liver failure if chemotherapy is commenced without antiviral therapy for hepatitis C.

(b) Viral serology is consistent with chronic active hepatitis $B$ infection and antiviral therapy should be given before chemotherapy is considered.

(c) Viral serology is consistent with previous exposure to hepatitis $B$ and $C$ and the patient will require antiviral prophylaxis to prevent hepatitis $B$ reactivation during immunosuppressive chemotherapy.

(d) Viral serology is consistent with previous hepatitis B infection, which is now cleared and no alterations to chemotherapy need to be considered.

(e) Viral serology is consistent with previous hepatitis B vaccination and no alterations to chemotherapy need to be considered.

3 A 32-year-old man with a three-year history of extensive ulcerative colitis presented to the accident and emergency department with a seven-day history of bloody diarrhoea. His regular medication included azathioprine. His stool frequency was eight times in the preceding 24 hours. On examination his temperature was $37.9^{\circ} \mathrm{C}$, heart rate was $105 \mathrm{bpm}$ and he was tender in the left iliac fossa. At flexible sigmoidoscopy he had continuous inflammation and ulceration extending beyond the limit of the examination. Histological 
examination of the rectal biopsies showed features of acute and chronic inflammation.

\section{What is the most appropriate treatment?}

(a) clarithromycin $500 \mathrm{mg}$ bd orally

(b) ganciclovir $5 \mathrm{mg} / \mathrm{kg}$ bd intravenously

(c) hydrocortisone $100 \mathrm{mg}$ qds intravenously

(d) metronidazole $400 \mathrm{mg}$ tds orally

(e) prednisolone $40 \mathrm{mg}$ od orally

4 A 28-year-old woman was initially treated for acute severe ulcerative colitis with intravenous hydrocortisone, prophylactic low molecular weight heparin and correction of hypokalaemia. She had a background history of congenital unilateral renal agenesis. On day three of treatment her stool frequency was 10 times over the preceding 24 hours, her haemoglobin was $98 \mathrm{~g} / \mathrm{L}$ (115-165), albumin 30g/L (35-50) and C-reactive protein $78 \mathrm{mg} / \mathrm{L}(<5)$. Abdominal $X$-ray showed a transverse colonic diameter of $4 \mathrm{~cm}$.

\section{What is the next most appropriate management step?}

(a) continue intravenous hydrocortisone for a total of 7 days

(b) CT abdomen

(c) intravenous ciclosporin $2 \mathrm{mg} / \mathrm{kg} / 24$ hours

(d) intravenous infliximab $5 \mathrm{mg} / \mathrm{kg}$

(e) subtotal colectomy

5 A 45-year-old man presented with a 12-hour history of sudden inability to swallow liquids or solids. Infrequent similar episodes over 10 years rarely lasted more than an hour. Endoscopy demonstrated an impacted meat bolus; after removal a slight narrowing was noted at the gastrooesophageal junction. Biopsies from the distal oesophagus showed 8 eosinophils per high power field, but none in proximal biopsies.

\section{Which one of the following options is correct?}

(a) eosinophilic oesophagitis is the most likely diagnosis

(b) initial treatment with oral budesonide is indicated

(c) oesophageal balloon dilation should be performed

(d) oesophageal manometry is indicated

(e) treatment with a proton pump inhibitor is likely to give long lasting improvement

6 A 60-year-old man with a history of epilepsy presented with severe chest pain radiating to the back coming on after a fit which happened an hour previously. He was found to be sweaty and short of breath. Pulse was $110 \mathrm{bpm}$. Chest and spinal X-rays were normal. Electrocardiogram showed T-wave inversion in the inferior and lateral chest leads.

\section{Which of the following statements are true?}

(a) chest CT scan should be performed as soon as possible

(b) lack of surgical emphysema in the neck makes aortic dissection likely

(c) non-ST segment elevation myocardial infarction is the most likely diagnosis

(d) oesophageal rupture is excluded by a normal chest X-ray

(e) suspicion of an oesophageal rupture should prompt immediate barium radiology.
7 Which one of the following is NOT a risk factor used in calculating the Rockall score?
(a) age
(b) cause of bleeding
(c) co-morbidity
(d) shock (haemodynamic instability)
(e) site of bleeding

8 A 70-year-old woman presented with haematemesis. Clinical examination demonstrated evidence of postural hypotension, tachycardia and melaena.

\section{What is the first step in the management of this patient?}
(a) intravenous access and fluid resuscitation
(b) intravenous erythromycin
(c) intravenous proton pump inhibitor infusion
(d) urgent surgical consultation
(e) urgent upper gastrointestinal endoscopy

9 A 45 year-old-man with a history of alcohol misuse attended the accident and emergency department with a minor scalp laceration following a fall; he was mildly intoxicated, confused but co-operative; his blood alcohol level was $320 \mathrm{mg} / 100 \mathrm{ml}$. His scalp laceration was cleaned and glued.

\section{What is the next step in the management of this patient?}
(a) give prophylactic oral thiamine supplementation
(b) move the patient to a side room so if he becomes violent he will be safe
(c) organise cerebral imaging
(d) prescribe benzodiazepines to prevent acute alcohol withdrawal
(e) refer to the alcohol liaison service

10 A malnourished 35-year-old woman undergoing medically assisted withdrawal from alcohol on the medical admissions unit became increasingly confused and agitated; her blood pressure was $105 / 65 \mathrm{mmHg}$ and her temperature had fallen to $35.3^{\circ} \mathrm{C}$.

\section{What is the most likely explanation for the findings?}
(a) inadequate control of her withdrawal syndrome
(b) ingestion/withdrawal from other psychoactive drugs
(c) intracerebral bleed
(d) occult gastrointestinal bleeding
(e) onset of Wernicke's encephalopathy

\section{CME Acute Medicine SAQ}

\section{Answers to the CME SAQ published in Clinical Medicine August 2015}

$\begin{array}{llllllllll}\text { Q1 } & \text { Q2 } & \text { Q3 } & \text { Q4 } & \text { Q5 } & \text { Q6 } & \text { Q7 } & \text { Q8 } & \text { Q9 } & \text { Q10 } \\ \text { (a) } & \text { (d) } & \text { (e) } & \text { (e) } & \text { (b) } & \text { (d) } & \text { (d) } & \text { (a) } & \text { (a) } & \text { (a) }\end{array}$

